\title{
COHERENT-STATE THEORY FOR THE PROTON-NEUTRON QUASISPIN GROUP ${ }^{\dagger}$
}

\author{
K. T. HECHT * \\ Physics Department, University of Michigan, Ann Arbor, Michigan 48109, USA \\ and \\ J. P. ELLIOTT
}

School of Mathematical and Physical Sciences, University of Sussex, Brighton, England

Received 5 November 1984

(Correction received 15 November 1984)

\begin{abstract}
An extended coherent-state theory ${ }^{1,2}$ ) is used to give a simple construction for the matrix elements of the proton-neutron sp(4) quasispin algebra. A very simple analytical expression is given for the matrix elements of the $s p(4)$ generators valid for all cases where initial and final states are multiplicity-free with respect to the $\mathrm{U}(1) \times \mathrm{SU}(2) n, T$ subalgebra. In the more general case, involving multiplicity, the most natural orthonormal basis constructed by the coherent-state method leads to many-nucleon states in the seniority scheme which are approximately labelled by $T_{p}$, the isospin of the $p$ nucleon pairs coupled to $J=0, T=1$.
\end{abstract}

\section{Introduction}

Recently an extended coherent-state theory has been developed by Rowe ${ }^{1}$ ) and in slightly different from by Deenen and Quesne $\left.{ }^{2}\right)^{\dagger \dagger}$ and applied to the noncompact symplectic groups and, in particular, the $\operatorname{Sp}(6, R)$ nuclear collective model ${ }^{3}$ ). A similar and detailed treatment of the $S p(4, R)$ group has been given by Castaños, Chacón, Moshinsky, and Quesne ${ }^{4}$ ) in their study of boson realizations of this group. Many of the compact groups of relevance for nuclear spectroscopy have also been discussed in terms of a generalized coherent-state theory by Dobaczewski ${ }^{5}$ ) in his functional representation analysis of boson expansion theories. Coherent-state expansions have also been used in the interacting boson model [for a recent example, see ref. $\left.^{6}\right)$ ]; but detailed applications of the generalized coherent-state methods to compact groups have so far lagged behind

\footnotetext{
† Work done at the Second Summer Institute in Theoretical Physics, Queen's University, Kingston, Canada, 1984.

* Supported in part by the US National Science Foundation.

${ }^{\dagger+}$ See refs. ${ }^{1,2}$ ) for a comprehensive listing of earlier references on coherent-state methods.
} 
the successful application made in the derivation of $\operatorname{Sp}(2 d, \mathrm{R})$ matrix elements. It is the purpose of this short note to show how the generalized coherent-state methods of refs. ${ }^{1,2,4}$ ) can be applied to advantage to compact groups with a specific simple example. The example chosen is that of the proton-neutron quasispin group, a unitary symplectic group in 4 dimensions, $S p(4)$, in its application to the $n, T$ structure of shell-model states in the seniority scheme, $(n=$ nucleon number, $T=$ isospin). A five-dimensional quasispin formalism (using the isomorphism between the Lie algebras $R(5)$ and $s p(4)$ ) has previously been developed ${ }^{7}$ ) to determine the $(n, T)$-dependent factors of shell-model matrix elements in the seniority scheme. Although a detailed practical solution to this problem has been found for most of the representations of interest in nuclear spectroscopy ${ }^{7}$ ), a "missing quantum number problem" has prevented a complete analytic solution. It will be shown how the extended coherent state methods can cope with this problem. It is of particular interest to note that the most natural orthonormal basis constructed by these methods is very closely related to a labeling scheme first proposed by $\operatorname{Racah}^{8}$ ). In this scheme a state of seniority $v$ and isospin $T$ is constructed by coupling the reduced isospin $t$ of the $v$ nucleons not coupled in pairs to $J=0$ with the resultant isospin $T_{p}$ of the $p$ pairs of nucleons coupled to $J=0, T=1$.

The coherent state realization of the $\mathrm{sp}(4)$ or proton-neutron quasispin algebra will be given in sect. 2 . The strategy used to calculate the matrix elements of this algebra is the same as that of refs. ${ }^{1,2}$ ). It uses coherent-state theory to relate the $\mathrm{sp}(4)$ algebra to a simpler algebra whose matrix elements are known. This simpler algebra generates a semi-direct product of a 3-dimensional Heisenberg-Weyl group with the $\mathrm{U}(1) \times \mathrm{SU}(2) n, T$ subgroup of $\mathrm{Sp}(4)$, where the Heisenberg-Weyl algebra is generated by Bargmann space variables $z_{i}$ and the adjoint differential operators $\partial / \partial z_{i}(i=1,2,3)$. As in ref. $\left.{ }^{1}\right)$ a very simple analytical expression can be derived for the matrix elements of the $\mathrm{sp}(4)$ generators valid for all cases where initial and final states are multiplicity-free with respect to the $U(1) \times S U(2)$ subalgebra, i.e. for all matrix elements involving only states which are completely specified by $n, T$, and $M_{T}$. In those cases where the additional fourth quantum number is needed the method involves the square root taking of an isoscalar, hermitean operator (or matrix). This case is illustrated in some detail with a few examples in sect. 3, with particular emphasis on the irreducible representation with seniority number $v=4$ and reduced isospin $t=2$. For nuclear shells with $j \geqslant \frac{11}{2}$ this involves states with threefold multiplicities. It is therefore beyond the scope of the earlier fivedimensional quasispin treatments but may have useful applications since configurations of both neutrons and protons in the $h_{\frac{1}{2}}$ abnormal-parity intruder orbit may play an important role in yrast-band spectroscopy of neutron deficient nuclei in the $A \sim 126$ mass region ${ }^{9}$ ). 


\section{Coherent-state realization of the proton-neutron quasispin group}

The proton-neutron quasi-spin group is generated by the nucleon pair-creation (and annihilation) operators coupled to $J=0, T=1, A^{+}\left(M_{T}\right)$ (and $A\left(M_{T}\right)$ ), the isospin generators $T$, and the number operator, or more conveniently $H_{1}=$ $\frac{1}{2} N_{\text {op }}-\left(j+\frac{1}{2}\right)$. [The notation follows that of ref. $\left.{ }^{7}\right)$.] It is convenient to introduce a vector notation $A^{+}=\left(A_{1}^{+}, A_{2}^{+}, A_{3}^{+}\right)$where $A^{+}\left(M_{T}= \pm 1\right)=\mp \sqrt{\frac{1}{2}}\left(A_{1}^{+} \pm i A_{2}^{+}\right)$, $A^{+}(0)=A_{3}^{+}$. The irreducible representations are labeled in terms of the seniority $v$ and reduced isospin $t$ by $\omega_{1}=j+\frac{1}{2}-\frac{1}{2} t$ and $t$. The states of an irreducible representation are spanned by vectors of the type

$$
\begin{gathered}
|\psi\rangle=\sum_{T_{p}} C_{T_{p}}\left|i^{n} \omega_{1}\left[t \times T_{p}\right] T M_{T}\right\rangle, \\
\left|j^{n} \omega_{1}\left[t \times T_{p}\right] T M_{T}\right\rangle=\sum_{M_{T_{p}}} \prod\left(A^{+}\right)_{p T_{p} M_{T_{p}}}\left|\omega_{1} t m_{t}\right\rangle\left\langle t m_{\imath} T_{p} M_{T_{p}} \mid T M_{T}\right\rangle,
\end{gathered}
$$

where $\prod\left(A^{+}\right)_{p T_{p} M_{T_{p}}}$ is a product of $p$ pair-creation operators coupled to resultant isospin $T_{p}$, with $p=\frac{1}{2}(n-v)$ and $T_{p}=p, p-2, \ldots$. The $\left|\omega_{1} t m_{\mathrm{r}}\right\rangle$ are the $v$-particle states $\left\langle j^{v} t m_{1}\right\rangle$. Note that $T_{p}$ is not an orthogonal label since the label $T_{p}$ cannot be associated directly with the eigenvalue of a hermitean operator. However, the states $\left.j^{n} \omega_{1}\left[t \times T_{\mathrm{p}}\right] T M_{T}\right\rangle$ are normalized. A generalized coherent state can be defined by

$$
\left|z, \omega_{1} t\right\rangle=\exp \left(z^{*} \cdot \boldsymbol{A}^{+}\right)\left|\omega_{1} t\right\rangle
$$

where $z=\left(z_{1}, z_{2}, z_{3}\right)$ defines three independent complex variables $z_{j}$. In eq. (2) $\left|\omega_{1} t\right\rangle$ is a shorthand notation and stands for the subspace of $(2 t+1)$ states $\left|\omega_{1} t m_{t}\right\rangle$ with $T=t$ and $n=v$. The states (2) are therefore a set of partially coherent states in the language of ref. ${ }^{2}$ ). Since all final-state vectors will be vector-coupled in isospin space (as in eq. (1)) and all matrix elements to be derived will be isospin reduced matrix elements, this distinction can be suffused in the shorthand notation $\left|\omega_{1} t\right\rangle$. The $|\psi\rangle$ thus have the functional representations

$$
\psi_{\omega_{1} i}(z)=\left\langle\omega_{1} t|\exp (z \cdot A)| \psi\right\rangle
$$

and operators $\mathcal{O}$ are represented by $\Gamma(\mathcal{O})$

$$
\begin{aligned}
\Gamma(\mathcal{O}) \psi_{\omega_{1} t}(z) & =\left\langle\omega_{1} t\left|\mathrm{e}^{(z \cdot A)} \mathcal{O}\right| \psi\right\rangle \\
& =\left\langle\omega_{1} t\left|\left(\mathcal{O}+[(z \cdot A), \mathcal{O}]+\frac{1}{2}[(z \cdot A),[(z \cdot A), \mathcal{O}]]+\ldots\right) \mathrm{e}^{(z \cdot A)}\right| \psi\right\rangle .
\end{aligned}
$$

This leads to the coherent-state realization

$$
\Gamma(A)=\frac{\partial}{\partial z}=\nabla
$$




$$
\begin{gathered}
\Gamma\left(\boldsymbol{A}^{+}\right)=\omega_{1} z-i[z \times t]-z(z \cdot \nabla)+\frac{1}{2}(z \cdot z) \nabla, \\
\Gamma\left(H_{1}\right)=-\omega_{1}+(z \cdot \nabla), \\
\Gamma(T)=t-i[z \times \nabla],
\end{gathered}
$$

where $z \cdot \nabla=\sum_{i} z_{j} \partial / \partial z_{j}$. The isospin operator acting on the implied label $m_{t}$ in the space of the $\psi_{\omega_{1}}$ with $n=v$ and isospin $t$ is denoted by $t$, an "intrinsic" isospin operator, which is combined with the "orbital" isospin operator $-i[z \times \nabla]$ in eq. (5d).

The operators

$$
\frac{\partial}{\partial z}, \quad z, \quad-\omega_{1}+(z \cdot \nabla), \quad t-i[z \times \nabla]
$$

generate a semi-direct product of a three-dimensional Heisenberg-Weyl group with a $U(1) \times S U(2)$ subgroup of $\operatorname{sp}(4)$; and the operators $\partial / \partial z$ and $z$ are a realization of a set of three-dimensional boson annihilation and creation operators $a$ and $a^{+}$. The states generated by these operators in the functional representation (3) (to be denoted by round brackets) for a number $p$ of bosons,

$$
\left.\left.\mid n \omega_{1}\left[t \times T_{p}\right] T M_{T}\right)=\sum_{M_{T_{p}}} P_{p T_{p} M_{T_{r}}}(z) \mid \omega_{1} t m_{t}\right)\left\langle t m_{t} T_{p} M_{T_{p}} \mid T M_{T}\right\rangle
$$

have here been expressed in terms of normalized $z$-space three-dimensional harmonic-oscillator functions :

$$
P_{p T_{p} M_{T_{p}}}(z)=\mathscr{N}_{p T_{p}}(z \cdot z)^{\frac{1}{2}\left(p-T_{p}\right)} \mathscr{Y}_{T_{p} M_{T_{p}}}(z)
$$

where $y$ is a solid harmonic in $z$; and $p=\frac{1}{2}(n-v)$. Note, however, that these are not the oscillator wave functions in real three-space because of the different definition of the scalar product in complex $z$-space [see ref. $\left.\left.{ }^{10}\right)\right]$. Instead, the functions (8) are the Bargmann transforms of the familiar three-dimensional harmonic-oscillator functions.

The reduced matrix elements of $z$ are given by standard Racah algebra:

$$
\left(n+2, \omega_{1}\left[t \times T_{p}^{\prime}\right] T^{\prime}\|z\| n \omega_{1}\left[t \times T_{p}\right] T\right)=U\left(t T_{p} T^{\prime} 1 ; T T_{p}^{\prime}\right)\left(T_{p}^{\prime}\|z\| T_{p}\right)
$$

where the $z$-space reduced matrix element follows from the simple properties ${ }^{10}$ ) of the $P_{p T_{p} M_{T_{p}}}(z)$ and is given by

$$
\left(T_{p}^{\prime}\|z\| T_{p}\right)=[(p+1)]^{\frac{1}{2}}\left\langle(p 0) T_{p} ;(10) 1 \|(p+1,0) T_{p}^{\prime}\right\rangle,
$$


with $T_{p}^{\prime}=T_{p} \pm 1$. The double-barred coefficient in (9b) is an $\mathrm{SU}(3) \supset \mathrm{R}(3)$ reduced Wigner coefficient of very simple algebraic form; see, e.g. ref. ${ }^{11}$ ), also eq. (20c) below. (It should be pointed out that the order of the vector couplings in eqs. (1) and (7) has been chosen to be $\left[t \times T_{p}\right]$. Also, the isospin reduced matrix elements of eq. (9a) are defined as follows: full matrix element-(reduccd matrix element $) \times($ Wigner coefficient $)$, without dimensional factors of $\left[2 T^{\prime}+1\right]^{\frac{1}{2}}$.)

Since $\nabla_{j}=\partial / \partial z_{j}$ is the hermitean adjoint of $z_{j}$ the realization (6) is a unitary realization of the $\mathrm{U}(1) \times \mathrm{SU}(2)$ Heisenberg-Weyl algebra, whereas the realization $\Gamma$, eqs. (5), of the $\mathrm{sp}(4)$ generators, is nonunitary. To evaluate the matrix elements of the $\mathrm{sp}(4)$ generators, we follow refs. ${ }^{1.2}$ ) [adhering to the notation of ref. ${ }^{1}$ )], and make a transformation to a unitary realization, $\gamma$, of the $\mathrm{sp}(4)$ generators by means of a hermitean positive definite $[\mathrm{U}(1) \times \mathrm{SU}(2)]$-invariant operator $\kappa$

$$
\gamma\left(A^{+}\right)=\kappa^{-1} \Gamma\left(A^{+}\right) \kappa, \quad \gamma(A)=\kappa^{-1} \Gamma(A) \kappa .
$$

The unitary requirement, $\gamma\left(A^{+}\right)=(\gamma(A))^{+}$, and eq. (5a) then give

$$
\Gamma\left(A^{+}\right)=\kappa^{2} z \kappa^{-2} .
$$

To determine $\kappa$ [following ref. $\left.\left.{ }^{1}\right)\right]$ we seek an $[\mathrm{U}(1) \times \mathrm{SU}(2)]$-invariant operator $A$ with the property

$$
[A, z]=\Gamma\left(A^{+}\right)
$$

Since the number of scalars which can be formed from the vectors $z, \nabla, t$ is limited, it is easy to show that the operator

$$
A=-\frac{1}{2}(z \cdot \nabla)(z \cdot \nabla)+\frac{1}{4}(z \cdot z) \nabla^{2}+\left(\omega_{1}+\frac{1}{2}\right)(z \cdot \nabla)+i t \cdot[z \times \nabla]
$$

is an operator with the necessary properties. It is diagonal in the basis (7) with eigenvalue $\Omega$,

$$
\begin{aligned}
\Omega(p & \left.=\frac{1}{2}(n-v), T_{p}, T\right) \\
& =-\frac{1}{4} p(p-1)+p\left(\omega_{1}+\frac{1}{2}\right)-\frac{1}{2} T(T+1)+\frac{1}{2} t(t+1)+\frac{1}{4} T_{p}\left(T_{p}+1\right),
\end{aligned}
$$

which follows from the simple action of operators $(z \cdot \nabla), \ldots$, on the $P_{p T_{p} T_{p}}(z)$. With these eigenvalues, the combination of eqs. (11) and (12),

$$
[\Lambda, z]=\kappa^{2} z \kappa^{-2},
$$

leads to recursion relations for the matrix elements of the operator $\kappa^{2}$. Since $\kappa^{2}$ is an $[\mathrm{U}(1) \times \mathrm{SU}(2)]$-invariant operator its matrix elements are diagonal in $n, T$ and 
independent of $M_{T}$ :

$$
\left(n^{\prime} \omega_{1}\left[t \times T_{p}^{\prime}\right] T^{\prime} M_{T}^{\prime}\left|\kappa^{2}\right| n \omega_{1}\left[t \times T_{p}\right] T M_{T}\right)=\delta_{n^{\prime} n} \delta_{T^{\prime} T} \delta_{M_{T}^{\prime} M_{T}}\left(\kappa^{2}\left(\omega_{1}, t, n, T\right)\right)_{\Gamma_{p}^{\prime} T_{p}}
$$

The dimension of the $\kappa^{2}$ matrix is thus given by the multiplicity of possible $T_{p}$ values for a state of definite $n$ and $T$; and this is generally a very small number. There are a large number of states in the seniority scheme in which the label $T_{p}$ is uniquely determined by the values of $v, t, n$, and $T$. Then $\kappa^{2}$ is 1 -dimensional. This includes all states ${ }^{7}$ ) of the irreducible representations with $t=0, t=\frac{1}{2}$ and with $\omega_{1}=t$, also all states with $n$ neutrons and no protons, with $(n-1)$ neutrons and one proton, and states with $t=1$ and $\frac{1}{2}(n-v)-T=$ even. The occurence of these simple (1-dimensional) states is therefore quite common so that this 1-dimensional case is of considerable interest. For the 1-dimensional case the square root taking of $\kappa^{2}$ required for eq. (10) is trivial. Taking matrix elements of relation (15) between simple (1-dimensional) states of type (7) with $n, T$ on the right and $n^{\prime}=n+2, T$ on the left yields

$$
\frac{\kappa^{2}\left(\omega_{1}, t, n+2, T^{\prime}\right)}{\kappa^{2}\left(\omega_{1}, t, n, T\right)}=\Omega\left(n+2, T_{p}^{\prime}, T^{\prime}\right)-\Omega\left(n, T_{p}, T\right)
$$

where it is assumed that $T_{p}$ (similarly $T_{p}^{\prime}$ ) is uniquely specified by $n$ and $T$. For such states the combination of eqs. (10), (11), and (17) gives

$$
\begin{aligned}
\left(n+2, \omega_{1}\left[t \times T_{p}^{\prime}\right] T^{\prime} \|\right. & \left.\gamma\left(A^{+}\right) \| n \omega_{1}\left[t \times T_{p}\right] T\right) \\
= & {\left[\Omega\left(n+2, T_{p}^{\prime}, T^{\prime}\right)-\Omega\left(n, T_{p}, T\right)\right]^{\frac{1}{2}} } \\
& \times\left(n+2, \omega_{1}\left[t \times T_{p}^{\prime}\right] T^{\prime}\|z\| n \omega_{1}\left[t \times T_{p}\right] T\right) .
\end{aligned}
$$

Since the carrier space spanned by the bosonic states (7) in the functional representation is mapped isomorphically into the fermion states $(1 \mathrm{~b})$, the matrix elements of the generators of the proton-neutron quasispin group in the fermion basis are also given by [cf. ref. $\left.\left.{ }^{1}\right)\right]$

$$
\begin{aligned}
&\left\langle j^{n+2} \omega_{1}\left[t \times T_{p}^{\prime}\right] T^{\prime}\left\|A^{+}\right\| j^{n} \omega_{1}\left[t \times T_{p}\right] T\right\rangle \\
&= {\left[\Omega\left(n+2, T_{p}^{\prime}, T^{\prime}\right)-\Omega\left(n, T_{p}, T\right)\right]^{\frac{1}{2}} } \\
& \times\left(n+2, \omega_{1}\left[t \times T_{p}^{\prime}\right] T^{\prime}\|z\| n \omega_{1}\left[t \times T_{p}\right] T\right) .
\end{aligned}
$$

This leads to a very simple general analytic expression for matrix elements of $A^{+}$ for the case when both initial and final states are simple (when both $T_{p}$ and $T_{p}^{\prime}$ are uniquely determined). In this case

$$
\begin{aligned}
\left\langle j^{n+2} \omega_{1}[t \times\right. & \left.\left.T_{p}^{\prime}\right] T^{\prime} \| A^{+}|| j^{n} \omega_{1}\left[t \times T_{p}\right] T\right\rangle \\
= & {\left[\Omega\left(n+2, T_{p}^{\prime}, T^{\prime}\right)-\Omega\left(n, T_{p}, T\right)\right]^{\frac{1}{2}} } \\
& \times[(p+1)]^{\frac{1}{2}}\left\langle(p 0) T_{p} ;(10) 1 \|(p+1,0) T_{p}^{\prime}\right\rangle U\left(t T_{p} T^{\prime} 1 ; T T_{p}^{\prime}\right),
\end{aligned}
$$


where

$$
\begin{aligned}
& \Omega\left(n+2, T_{p}^{\prime}, T^{\prime}\right)-\Omega\left(n, T_{p}, T\right) \\
& \quad=\frac{1}{2}\left[2 \omega_{1}+1-\frac{1}{2}(n-v)+T(T+1)-T^{\prime}\left(T^{\prime}+1\right)-\frac{1}{2} T_{p}\left(T_{p}+1\right)+\frac{1}{2} T_{p}^{\prime}\left(T_{p}^{\prime}+1\right)\right]
\end{aligned}
$$

and, with $p=\frac{1}{2}(n-v)$

$$
\begin{aligned}
{[(p+1)]^{\frac{1}{2}}\left\langle(p 0) T_{p} ;(10) 1 \|(p+1,0) T_{p}^{\prime}\right\rangle } & \begin{cases}{\left[\frac{\left(T_{p}+1\right)\left(\frac{1}{2}(n-v)+T_{p}+3\right)}{\left(2 T_{p}+3\right)}\right]^{\frac{1}{t}}} & \text { for } T_{p}^{\prime}=T_{p}+1 \\
-\left[\frac{T_{p}\left(\frac{1}{2}(n-v)-T_{p}+2\right)}{\left(2 T_{p}-1\right)}\right]^{\frac{1}{3}} & \text { for } T_{p}^{\prime}=T_{p}-1_{*}\end{cases}
\end{aligned}
$$

This result is in agreement with all the 1-dimensional cases included in ref. ${ }^{7}$ ) but is of wider applicability.

\section{Non-simple cases}

For states which are not free of multiplicity with respect to the $\mathrm{U}(1) \times \mathrm{SU}(2)$ subalgebra the $\kappa^{2}$ operator can be represented in matrix form, (16). Its matrix elements can be evaluated recursively from simple simultaneous equations by putting eq. (15) into the form

$$
\kappa^{2} z=[A, z] \kappa^{2}
$$

or

$$
\begin{aligned}
\sum_{T_{p}^{\prime \prime}} \kappa^{2}\left(n+2, T^{\prime}\right)_{T_{p}^{\prime} T_{p}^{\prime}}\left(n+2, \omega_{1}\left[t \times T_{p}^{\prime}\right] T^{\prime} \| z \mid n \omega_{1}\left[t \times T_{p}\right] T\right) \\
=\sum_{\bar{T}_{p}}\left[\Omega\left(n+2, T_{p}^{\prime}, T^{\prime}\right)-\Omega\left(n, \bar{T}_{p}, T\right)\right] \\
\\
\quad \times\left(n+2, \omega_{1}\left[t \times T_{p}^{\prime}\right] T^{\prime}\|z\| n \omega_{1}\left[t \times \bar{T}_{p}\right] T\right) \kappa^{2}(n, T)_{T_{p} T_{p}}
\end{aligned}
$$

The required square root of the $\kappa^{2}$ operator can then be taken if $\kappa^{2}$ is diagonalized via

$$
\sum_{T_{p} T_{p}} U_{i T_{p}}^{-1}\left(k^{2}\right)_{T_{p} T_{p}} U_{\left.T_{p}\right)}=\lambda_{i} \delta_{i j}
$$


For non-simple states the matrix elements of the $\mathrm{sp}(4)$ generators are then given by

$$
\begin{aligned}
\left\langle j^{n+2} \omega_{1} t i T^{\prime}\left\|\boldsymbol{A}^{+}\right\| j^{n} \omega_{1} t k T\right\rangle & \\
= & {\left[\lambda_{i}^{\prime}\left(n+2, T^{\prime}\right)\right]^{\frac{1}{2}} \sum_{T_{p}^{\prime} T_{p}} U_{T_{p}^{\prime}}^{\prime *}\left(n+2, \omega_{1}\left[t \times T_{p}^{\prime}\right] T^{\prime}\|z\| n \omega_{1}\left[t \times T_{p}\right] T\right) } \\
& \times U_{T_{p} k}\left[\lambda_{k}(n, T)\right]^{-\frac{1}{2}},
\end{aligned}
$$

where the reduced matrix element of $z$ is given by eq. (9), and $i=1, \ldots d$, $k=1, \ldots, d$. Here $d^{\prime}$ and $d$ are the multiplicities of the states with $n+2, T^{\prime}$ and $n, T$, respectively. Since eq. (22) can be followed by a further arbitrary unitary transformation, after square-root taking, there is nothing unique about the states $k=1, \ldots d$. However, for the coherent state theory they will be considered as the "most natural" orthonormal basis states. Surprisingly, the states of this "most natural" basis each has a large overlap with a particular value of $T_{p}$. This is now illustrated with some examples.

To compare with the earlier solutions ${ }^{7}$ ) the first example chosen is that of the irreducible representation $\omega_{1}, t=1$. For this representation states with $\frac{1}{2}(n-v)-T=$ even integer $=2 k$ are simple; with $T_{p}=T$, uniquely specified. For these states a recursive application of eq. (17) gives

$$
\frac{\kappa^{2}(n, T)}{\kappa^{2}(v, t)}=\left(\omega_{1}+1\right) \frac{\left(\omega_{1}-1\right) ! I\left(\omega_{1}+\frac{3}{2}\right)}{\left(\omega_{1}-k-T\right) ! \Gamma\left(\omega_{1}+\frac{3}{2}-k\right)}, \quad k=0,1, \ldots
$$

Without loss of generality the $\kappa^{2}$ value for the lowest weight state $\kappa^{2}(v, t)$ will be replaced by unity. States in the irreducible representation $\omega_{1}, t=1$ with $\frac{1}{2}(n-v)-T=$ odd integer, in general, have a 2 -fold multiplicity. For these states recursive application of eqs. of type (21) give, with $\frac{1}{2}(n-v)=T+2 k+1$; $k=0,1,2, \ldots$ :

$$
\left(\kappa^{2}(n, T)\right)_{T_{p}^{\prime} T_{p}}=\frac{\left(\omega_{1}-1\right) ! \Gamma\left(\omega_{1}+\frac{3}{2}\right)}{\left(\omega_{1}-k-T\right) ! \Gamma\left(\omega_{1}+\frac{3}{2}-k\right)} \frac{1}{2(2 T+1)} M_{T_{p}^{\prime} T_{p}}
$$

where

$$
\begin{aligned}
& M_{T-1, T-1}=\left\{\left(\omega_{1}+1\right)\left(2 \omega_{1}+1-2 k\right)+T\left(2 \omega_{1}+3\right)\left(2 \omega_{1}-1-2 k\right)-2 T^{2}\left(2 \omega_{1}+3\right)\right\}, \\
& M_{T-1, T+1}=[2(k+1) T(T+1)(2 T+2 k+3)]^{\frac{1}{2}}, \\
& M_{T+1, T+1}=2\left\{\left(\omega_{1}+2\right)\left(\omega_{1}-k\right)+T\left[\left(2 \omega_{1}^{2}+4 \omega_{1}+1\right)-k\left(2 \omega_{1}+3\right)\right]\right\} .
\end{aligned}
$$

For this case the U-matrix for eq. (23) can be expressed in the form

$$
U=\left(\begin{array}{cc}
-\cos \theta & \sin \theta \\
\sin \theta & \cos \theta
\end{array}\right)
$$

We also note that for states on the boundary of allowed $n, T$-values; that is, for states with $T=\omega_{1}-k$, the $M$-matrix has zero determinant, leading to one zero 
eigenvalue $\lambda$, as required by the fact that these states are simple. (In this case the single allowed state corresponds to the second column of eq. (26), the one appropriate to the larger of the two eigenvalues, $\lambda$ ). In all practical $j$-shell cases of this type which have been considered, the orthonormal basis states corresponding to the larger (smaller) eigenvalue $\lambda$ are dominated by the larger (smaller) of the two possible $T_{p}$ values; that is $\cos \theta \sim 1$, so that the label $T_{p}$ has relevance in this "most natural" basis. This is illustrated with a specific example in table 1 which gives the $\cos \theta$ values for states with $j=\frac{11}{2}, v=2, t=1$. [The labels $\beta=1$ and 2 of ref. $^{7}$ ) can be regained by following eq. (23) with a further unitary transformation; but, as pointed out in ref. ${ }^{7}$ ), no special physical relevance can be assigned to these $\beta$-labels which were chosen largely for practical reasons of calculational simplicity.]

As a second example, we consider the irreducible representations $\omega_{1} t$, with $t=2$, which were beyond the scope of ref. $\left.{ }^{7}\right)$. In these irreducible representations states with $\frac{1}{2}(n-v)-T=$ odd integer $=(2 k+1)$, in general, have a twofold multiplicity. For these states (with $k=0,1,2, \ldots$ )

$$
\left(\kappa^{2}(n, T)\right)_{T_{p} T_{p}}=\frac{\left(\omega_{1}-2\right) !\left(\omega_{1}+1\right) \Gamma\left(\omega_{1}+\frac{3}{2}\right)}{\left(\omega_{1}-k-T\right) ! 2(2 T+1) \Gamma\left(\omega_{1}+\frac{3}{2}-k\right)} M_{T_{p}^{\prime} T_{p}}
$$

where

$$
\begin{aligned}
M_{T-1, T-1}= & \left\{\left(2 \omega_{1}^{2}+3 \omega_{1}+9\right)-2 k\left(\omega_{1}-3\right)\right. \\
& \left.+T\left(2 \omega_{1}+3\right)\left(2 \omega_{1}-1-2 k\right)-2 T^{2}\left(2 \omega_{1}+3\right)\right\}, \\
M_{T-1, T+1}= & 3[2(k+1)(T-1)(T+2)(2 T+2 k+3)]^{\frac{1}{2}}, \\
M_{T+1, T+1}= & 2\left\{\left(\omega_{1}^{2}+2 \omega_{1}-6\right)-k\left(\omega_{1}+6\right)+T\left[\left(2 \omega_{1}^{2}+4 \omega_{1}-3\right)-k\left(2 \omega_{1}+3\right)\right\} .\right.
\end{aligned}
$$

TABLE 1

The most natural basis for $j=\frac{11}{2}, v=2, t=1$

\begin{tabular}{rlllll}
\hline$n$ & $T$ & $\left.\cos \theta^{\mathrm{a}}\right)$ & $n$ & $T$ & $\cos \theta$ \\
\hline 6 & 1 & 0.99675 & 14 & 1 & 0.98134 \\
8 & 2 & 0.99833 & 16 & 2 & $0.99202^{\mathrm{b}}$ \\
10 & 3 & 0.99890 & 18 & 3 & $\left.0.99523^{\mathrm{b}}\right)$ \\
12 & 4 & 0.99919 & & & \\
14 & 5 & $\left.0.99936^{\mathrm{b}}\right)$ & 18 & 1 & 0.96937 \\
10 & 1 & 0.99055 & 20 & 2 & $0.98742^{\mathrm{b}}$ ) \\
12 & 2 & 0.99567 & 22 & & $\left.0.95513^{\mathrm{b}}\right)$ \\
14 & 3 & $\left.0.99731^{\mathrm{b}}\right)$ & & & \\
16 & 4 & $\left.0.99808^{\mathrm{b}}\right)$ & & & \\
\hline
\end{tabular}

a) See eqs. (26) and (23).

b) Maximal states with only one non-zero eigenvalue, $\lambda$. 
The first threefold multiplicity occurs for states with $\frac{1}{2}(n-v)-T=2$. For these the $\kappa^{2}$ matrix is given by

$$
\left(\kappa^{2}(n, T)\right)_{T_{p}^{\prime} T_{p}}=\frac{\left(\omega_{1}-2\right) !}{\left(\omega_{1}-T\right) !} \frac{1}{(2 T+1)} M_{T_{p}^{\prime} T_{p}},
$$

where

$$
\begin{aligned}
M_{T-2, T-2}= & \frac{1}{2(2 T-1)}\left\{\left(-2 \omega_{1}^{4}-6 \omega_{1}^{3}+\frac{25}{2} \omega_{1}^{2}+\frac{51}{2} \omega_{1}+9\right)\right. \\
& +T\left(4 \omega_{1}^{3}+38 \omega_{1}^{2}+31 \omega_{1}-\frac{51}{2}\right)+T^{2}\left(8 \omega_{1}^{4}+24 \omega_{1}^{3}-4 \omega_{1}^{2}-62 \omega_{1}-\frac{87}{2}\right) \\
& \left.+T^{3}\left(-16 \omega_{1}^{3}-56 \omega_{1}^{2}-28 \omega_{1}+30\right)+T^{4}\left(8 \omega_{1}^{2}+32 \omega_{1}+30\right)\right\} \\
M_{T-2, T}= & {\left[\frac{3}{2}(T-1)(T+1)(2 T+1) /(2 T-1)^{2}\right]^{\frac{1}{2}}\left\{3\left(\omega_{1}+1\right)\left(2 \omega_{1}+1\right)\right.} \\
& \left.+T\left(4 \omega_{1}^{2}-9\right)-2 T^{2}\left(2 \omega_{1}+3\right)\right\} \\
M_{T-2, T+2}= & 3[(T-1) T(T+1)(T+2)(2 T+5) / 2(2 T-1)]^{\frac{1}{2}}, \\
M_{T, T}= & {[(2 T+1) /(2 T-1)(2 T+3)]\left\{\left(-3 \omega_{1}^{4}-\frac{15}{2} \omega_{1}^{3}-24 \omega_{1}^{2}-\frac{57}{2} \omega_{1}-9\right)\right.} \\
& +T\left(4 \omega_{1}^{4}+13 \omega_{1}^{3}+\frac{11}{2} \omega_{1}^{2}+9 \omega_{1}+\frac{45}{2}\right) \\
& \left.+T^{2}\left(4 \omega_{1}^{4}+6 \omega_{1}^{3}-6 \omega_{1}^{2}+2 \omega_{1}+24\right)+T^{3}\left(-4 \omega_{1}^{3}-10 \omega_{1}^{2}-4 \omega_{1}+6\right)\right\} \\
M_{T, T+2}= & {\left[3 T(T+2)(2 T+1)(2 T+5) /(2 T-1)(2 T+3)^{2}\right]^{\frac{1}{2}}\left\{2 T \omega_{1}\left(\omega_{1}+2\right)\right.} \\
& \left.-\left(\omega_{1}^{2}+2 \omega_{1}+3\right)\right\}, \\
M_{T+2, T+2}= & {[1 /(2 T+3)]\left\{3\left(\omega_{1}-1\right) \omega_{1}\left(\omega_{1}+2\right)\left(\omega_{1}+3\right)+T\left(8 \omega_{1}^{4}+32 \omega_{1}^{3}\right.\right.} \\
& \left.\left.+10 \omega_{1}^{2}-44 \omega_{1}-12\right)+T^{2}\left(4 \omega_{1}^{4}+16 \omega_{1}^{3}+8 \omega_{1}^{2}-16 \omega_{1}-6\right)\right\} .
\end{aligned}
$$

In the special case, when $T=\omega_{1}$, this symmetric matrix has two zero eigenvalues, in agreement with the fact that this state is simple. Also, with $T=\omega_{1}-1$ there are only two nonzero eigenvalues, again in agreement with the fact that states one step inside the boundary of maximal $n, T$ values can have at most a twofold multiplicity. For states with $j=\frac{11}{2}, v=4, t=2$, for example, the only threefold multiplicity occurs for the single case $n=12, T=2$. In this case the $U$-matrix of eqs. (22) and (23) has the numerical value

$i=1$
$T_{p}=0$
$T_{p}=2$
$T_{p}=4$$\quad\left(\begin{array}{rrr}0.92848 & 0.37071 & 0.02225 \\ -0.37061 & 0.92107 & 0.11945 \\ 0.02379 & -0.11916 & 0.99259\end{array}\right]$


showing again that the three "most natural" states in the basis defined by the eigenvalues $\lambda_{1}<\lambda_{2}<\lambda_{3}$ are dominated by the values $T_{p}=0,2$, and 4 , respectively.

In conclusion, it can be stated that the extended coherent state theory gives a simple method of constructing the matrix clements of the proton-ncutron quasispin algebra, a compact $\mathrm{sp}(4)$ algebra. Moreover, the most natural orthonormal basis constructed by these methods for the proton-neutron quasispin algebra leads to many-nucleon states in the seniority scheme for which the label $T_{p}$ has significance. This label, which is natural to the associated $U(1) \times S U(2)$ Heisenberg-Weyl algebra thus plays a role very similar to that of the corresponding $U(3)$ labels in the coherent state theory of the noncompact $\operatorname{sp}(6, \mathbf{R})$ algebra of ref. $\left.{ }^{1}\right)$. As in the latter case, the off-diagonal matrix elements of the $\kappa^{2}$ operators could be neglected altogether in the limit $j \rightarrow \infty$; or, for fixed (large) $j, T \rightarrow j$. Although these limiting cases are now not generally valid in the practical applications, the extended coherent-state method nevertheless leads to a many-nuclcon seniority basis which is approximately labelled by $T_{p}$. Note, however, that a recent study ${ }^{12}$ ) of the physical significance of the label $T_{p}$ in the $\mathrm{f}_{\frac{7}{2}}$ shell indicates that $T_{p}$ is strongly mixed and that this mixing is close to that which corresponds to definite $U(6)$ symmetry in the interacting boson model.

The work reported in this investigation was carried out at the Second Summer Institute in Theoretical Physics at Queen's University, Kingston, Ontario, Canada as a result of discussions on symplectic symmetry and extended coherent-state theories. It is a pleasure, in particular, to acknowledge valuable discussions with D. J. Rowe, M. Moshinsky, C. Quesne, and G. Rosensteel, and to express our thanks to the Natural Sciences and Engineering Research Council of Canada and the organizers of the summer institute for their hospitality and the stimulating atmosphere of the institute.

\section{References}

1) D. J. Rowe, J. Math. Phys. 25 (1984) 2662;

D. J. Rowe, G. Rosensteel and R. Carr, J. of Phys. A17 (1984) L399

2) J. Deenen and C. Quesne, J. Math. Phys. 25 (1984) 2354; Université Libre de Bruxelles preprint PTM 84-07; J. of Phys. A17 (1984) L405

3) G. Rosensteel and D. J. Rowe, Phys. Rev. Lett. 38 (1977) 10; Ann. of Phys. 126 (1980) 343

4) O. Castaños, E. Chacón, M. Moshinsky and C. Quesne, to be published

5) J. Dobaczewski, Nucl. Phys. A369 (1981) 213 (I), 237 (II); A380 (1982) 1 (III)

6) G. Wenes, A. E. L. Dieperink and O. S. van Roosmalen, Nucl. Phys. A424 (1984) 81

7) K. T. Hecht, Phys. Rev. 139B (1965) 794; Nucl. Phys. A102 (1967) 11; and references therein;

R. P. Hemenger and K. T. Hecht, Nucl. Phys. A145 (1970) 468; and references therein

8) G. Racah, Proc. Rehovoth Conf. on nuclear structure, ed. H. J. Lipkin (North-Holland, Amsterdam, 1958)

9) K. J. Weeks, J. P. Draayer and K. T. Hecht, Nucl. Phys. A414 (1984) 42;

J. P. Draayer, C. S. Han, K. J. Weeks and K. T. Hecht, Nucl. Phys. A365 (1981) 127 
10) V. Bargmann, Comm. Pure Appl. Math. 14 (1961) $187 ; 20$ (1967) 1; see also P. Kramer, G. John and D. Schenzle, Group theory and the interaction of composite nucleon systems (Vieweg, Braunschwcig, 1981) ch. 4;

K. T. Hecht and YY. Suzuki, J. Math. Phys. 24 (1983) 785

11) J. D. Vergados, Nucl. Phys. A111 (1968) 681

12) J. A. Evans, J. P. Elliott and S. Szpikowski. Nucl. Phys. A435 (1985) 317 